\title{
The First 30 Days of COVID 19 Vaccination in Cameroon: Achievements, Challenges and Lessons Learned
}

Amani Adidja, MD $^{1,3}$, Djossaya Dove MPH ${ }^{2}$, André Bita ${ }^{4}$

${ }^{1}$ Sub-directorate of Vaccination, Directorate of Family Health, Cameroon Ministry of Public Health Cameroon; amaniadidja@gmail.com

${ }^{2}$ Health Research Econometrics National Institute of Statistics Cameroon , djossayadove@gmail.com

${ }^{3}$ Faculty of Medicine and Biomedical Sciences, University of Yaoundé I, Yaoundé, Cameroon ${ }^{4}$ World Health Organization Afro UCN/VPD

*Corresponding Author: Adidja Amani MD MPH PhDc, Deputy Director for Vaccination, Ministry of Public Health -Yaoundé-Cameroon; Tel: +237662603240; E-mail: amaniadidja@gmail.com

'These authors contributed equally to this work'

Key-words: vaccination, the first 30 days; COVID-19 vaccines introduction; Cameroon; achievements; challenges; lessons learned 


\begin{abstract}
Cameroon's national vaccination campaign was launched on April 12, 2021, amid a nationwide outbreak of COVID-19 with two types of vaccines. This study provides preliminary evidence of the level of coverage of the population and gives an early overview of the challenges, the achievements and the lessons learned.

COVID-19 vaccine administration data were obtained from data of the Cameroon Ministry of Public Health. Descriptive statistical analyses were conducted.

Thirty days after the introduction of COVID 19 vaccines, five percent of the target population was vaccinated. Women represented one third of the people vaccinated regardless of age and health conditions. Although AEFI reported were minor and scanty with both vaccines, the majority of the vaccinated did not come back for their second dose. There is a need to build confidence among eligible beneficiaries in order to expand the benefits of vaccination to control the current pandemic.

The country is still far below the target which could be worrisome given that the uptake is slow and, the 391200 doses of the AstraZeneca are going to expire in August 2021. This study offers insights into those early efforts as a contribution to significant discussions about upcoming approach to improve service delivery and uptake.
\end{abstract}

\title{
Funding
}

None 


\section{Background}

The resurgence of Covid-19 cases and the occurrence of a new wave of contaminations in Cameroon, between February 25 and March 3, 202 ${ }^{1}$, arose at a time when the scientific community had a new response to this pandemic, the vaccination which is being introduced in many countries.

The National Immunization Technical Advisory Groups (NITAG) and the Scientific Committee for COVID19 responses prioritized the target populations for the vaccination against Covid-19 according to as follows: front-line health and social workers, people over 50 with morbidities or conditions, workers over 50 years who are critical for the functioning of the State; refugees over 50, staff from Embassies and diplomatic missions accredited to the Cameroon, people under the age of 50 with comorbidities with significant higher risk of serious illness or death, eligible refugees under the age of 50, Other people over 50 years old. In addition workers under 50 years old critical functioning of the State (Government, administrative bodies, Parliament, judiciary, regional councils, municipal councilors), eligible teachers, students and pupil not taken into account in previous group; and finally other target groups like travelers, transporters, prisoners, refugees, and other basic social sectors. ${ }^{2}$

On Sunday evening of April 11, 2021, the Prime Minister of Cameroon received a donation of 200,000 doses of Sinopharm from the Chinese Government. As the country was eligible for the Advance Market Commitment 92 financing mechanism of the COVAX facility ${ }^{3}$, on April $17^{\text {th }}$ the country received 391,200 doses out of the 1,200,000 doses awaited of AstraZeneca vaccines. The country aims to vaccinate 5,400,000 people against Covid-19, by the end of 2021, then 15 million Cameroonians in 2022 in order to reach the threshold vaccination coverage which confers herd immunity. 4

Cameroon has launched the deployment of vaccination against Covid-19 on April $12^{\text {th }}$ by the Minister of Public Health in the capital of the country, at the specialized center for COVID-19 patients of the Yaoundé Central hospital. Following implementation of any new vaccination program, an evaluation of its coverage, effectiveness, and safety in real-life setting is essential as the first 30 days of a new vaccination program are crucial to establishing the routines. . This paper intends to assess early on the level of implementation of the vaccination response strategy. 


\section{Methods}

\section{Target setting and population estimates}

The Republic of Cameroon, is a country in Central Africa, which stretches from the Gulf of Guinea to the Lake Chad. Its particular geographical location explains the diversity of its climate (equatorial and tropical) and its natural landscape. Cameroon has an area of $475,650 \mathrm{~km}^{2}$. It is limited to the east by the Republic Central African, in the North-East by the Republic of Chad, in the South by the Republic of Congo, the Republic of Gabon and the Republic of Equatorial Guinea, in the southwest by the Atlantic Ocean and west by the Federal Republic of Nigeria.

\section{Demographic situation}

Based on demographic projections ${ }^{5}$, the population of Cameroon is estimated at approximately $27,142,557$ inhabitants with a median age of 18.7 years.

\section{Data availability}

COVID-19 vaccine administration data were obtained from the Cameroon Ministry of Public Health data. Daily data are compiled from the COVID-19 individual vaccination record from the 244 vaccination sites. Although the data management system will gradually be automated, the vaccination register has the contact of the vaccinated person, the characteristics of the vaccinated person that are necessary (sex, age, occupational group, risk profile, etc.).

All the data and statistical code to reproduce the tables and figures in the manuscript and Supplementary Information are published daily by the Ministry of Public Health of Cameroon. The data that support the findings of this study are available on request from the corresponding author. Descriptive statistical analyses were conducted on Excel 5.0.

\section{RESULTS}

\section{Achievements}

The first month of the introduction has been filled with activities, which were supposed to be implemented before the vaccine's arrival in the country. It zas mainly capacity building including Cascade training workshops including AEFI, deployment of electronic forms for data 
management, gradual opening of vaccination centers, training of trainers at different levels, and seminars for healthcare workers within the framework of Africa Immunization week.

\section{- Vaccine uptake and Coverage}

The vaccine uptake is the number of people vaccinated with a certain dose of the vaccine in a certain time period. At the time of writing, two types of vaccines were administered in Cameroon namely Sinopharm and covidshield. 43,651 people have received their first dose and 1,061 have already received their second dose of vaccine against Covid-19 which represents 5.4 percent and less than 1 percent respectively of the target population. The First dose AstraZeneca stands at $2.18 \%$ against $3.18 \%$ for Sinopharm.

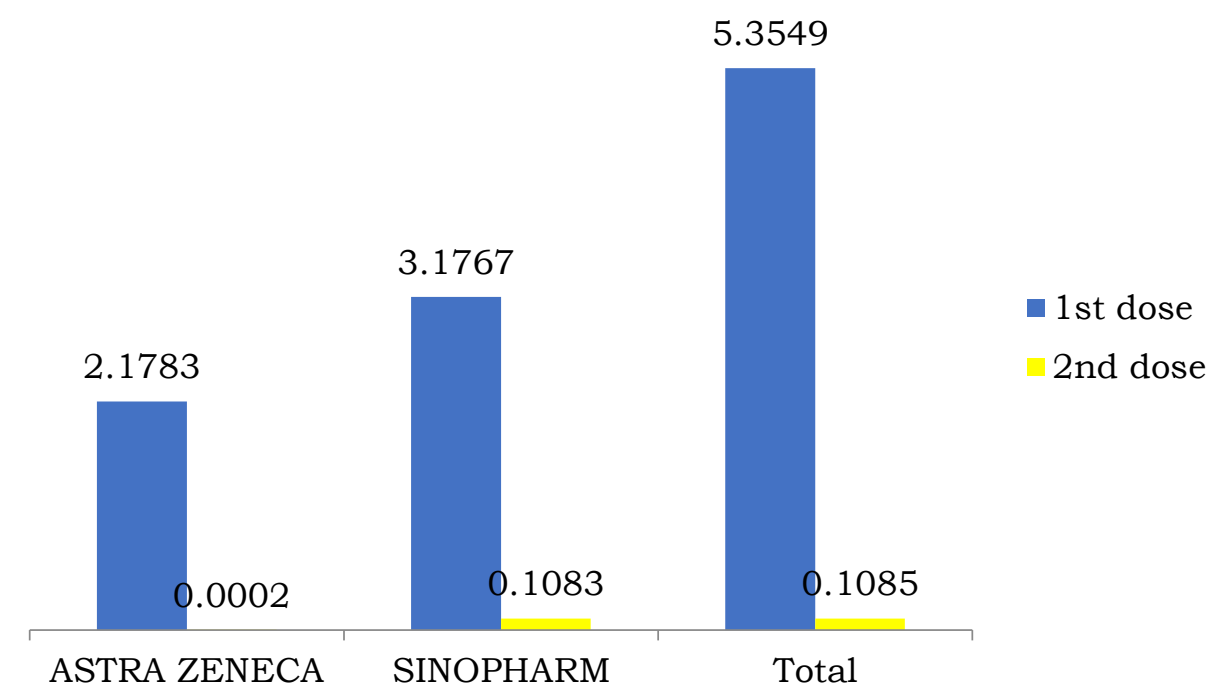

Figure 1: Coverage (in percentage \%) by dose of each vaccine from 12 April to 11 May 2021

\section{- Progress in implementation}

After a gradual increase from April 12, the date of the official launch until May 3, there has been a stagnation of coverage over the last 7 days. Progressive implementation from districts on the first week to all the district at the end of the first month; 177 out of 190; that is a performance 93 per cent are implementing vaccination Steps of new vaccine introduction planning has been accelerated to ensure optimal uptake across the country. 


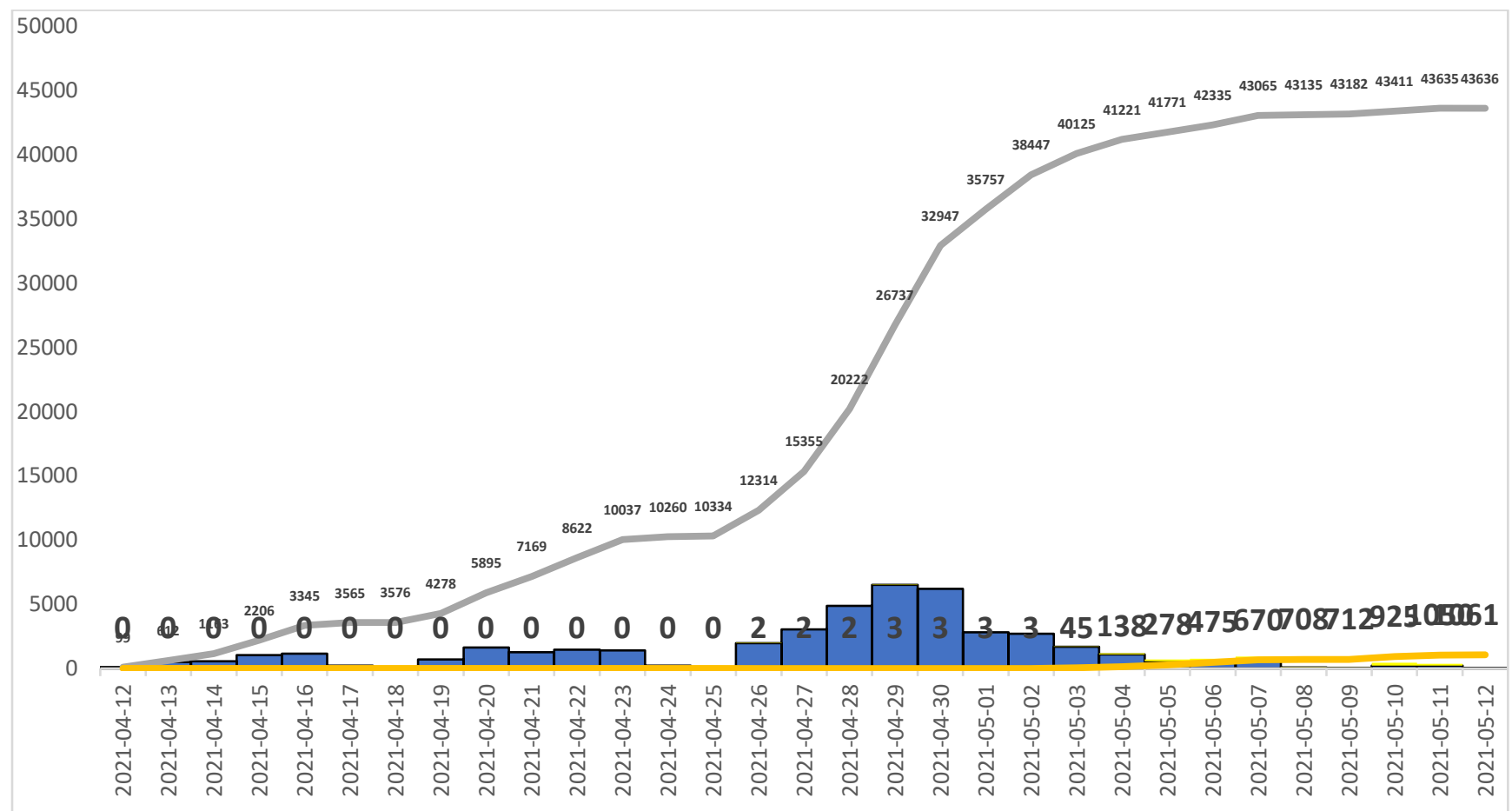

Figure 2 daily progress of doses administered by types of covid19 vaccines on the first 30 days in Cameroon source Daily progression of increase uptake of vaccines

\section{- Distribution by priority groups and by gender}

For both Sinopharm and AstraZeneca, the distribution by priority groups shows that health personnel represent 30 percent of the vaccinated population, and less than 12 percent of the vaccinated group presented comorbidities. Old people age 50 years and women represented above represent less than 31 and 32,5 percent respectively. 


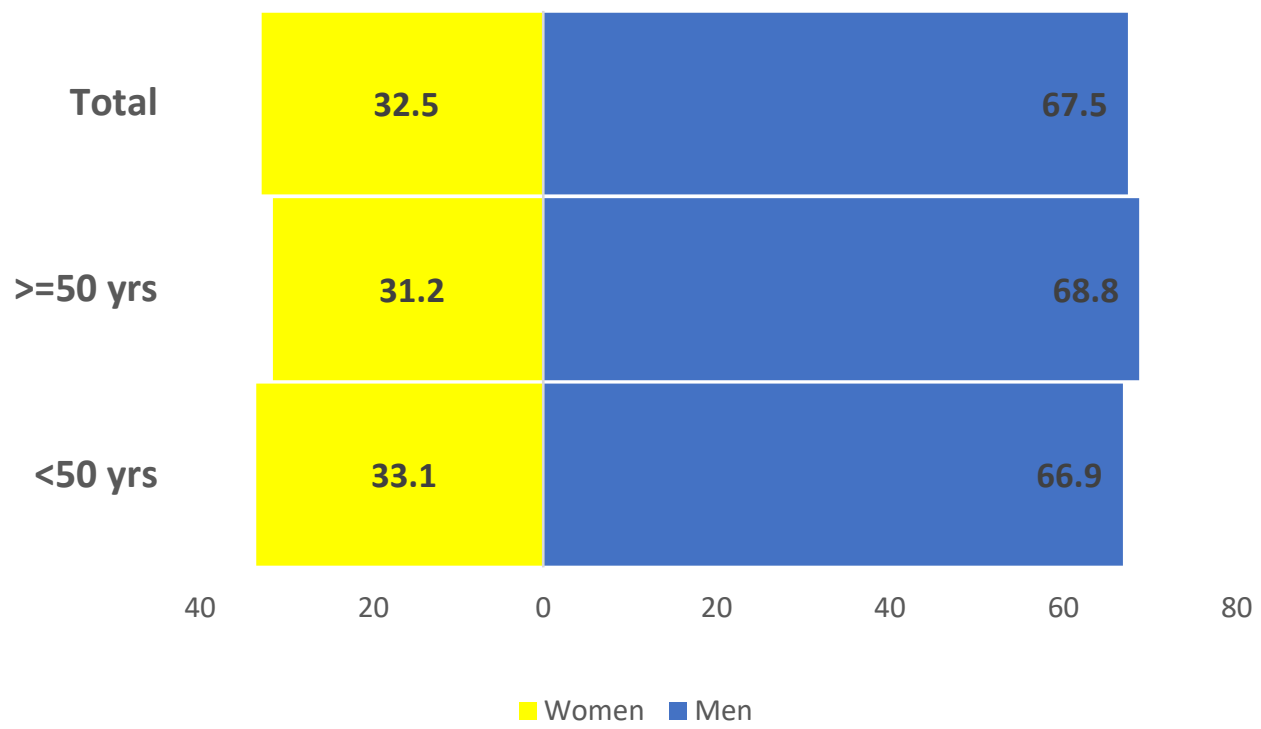

Figure 3: Distribution (in \%) of persons vaccinated by age group and sex

\section{- Vaccine safety}

As with any vaccine roll-out, it is very important to monitor, record, investigate, and reporting any adverse events following immunization. 77 minor cases that is 0,17 percent reported and six severe cases of adverse events following vaccination were reported. More specifically, for AstraZeneca, out of 26,695 doses administered, 19 minor cases and 1 severe AEFI cases were reported as against 58 and 5 cases respectively for Sinopharm out of a total of 17,696 doses. Among the side effects reported, the most frequently reported were headache, fever and myalgia with respective rate of 35, 25 and 16 per cent .

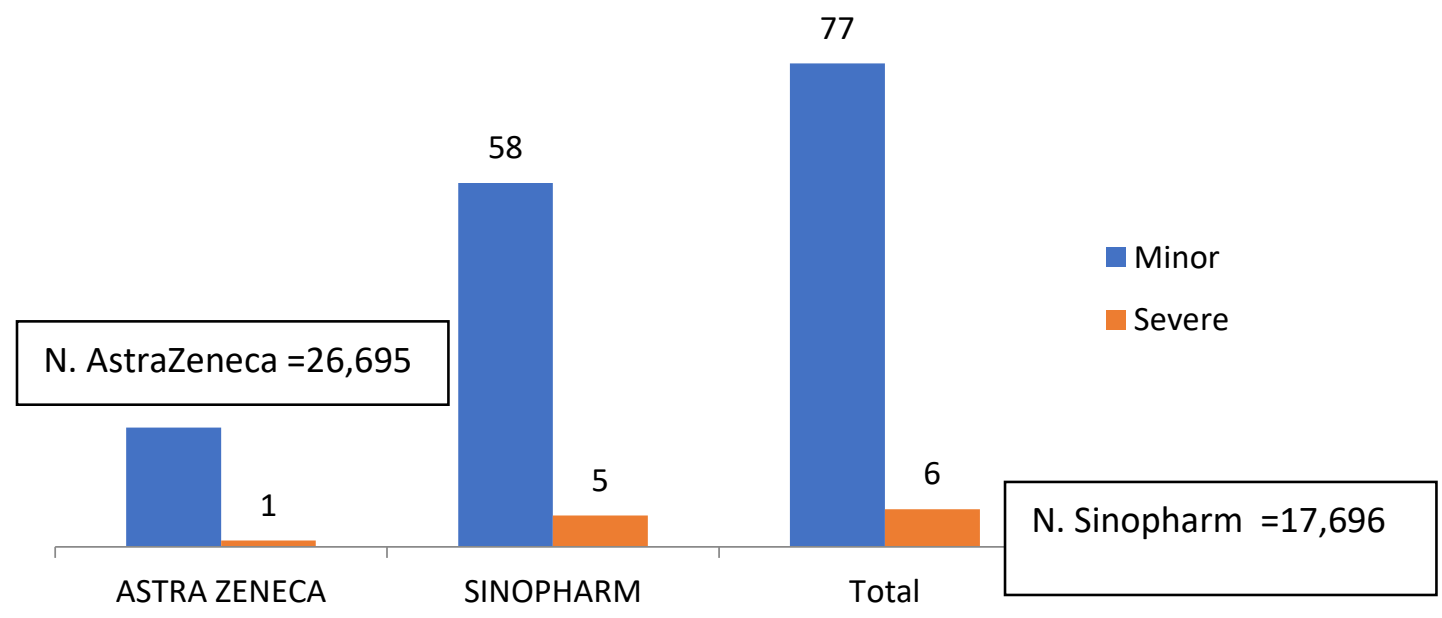


Figure 4: Number of AEFI cases by vaccine type and form

\section{- Vaccination timeliness of the second dose}

The percentage of people who received at least one dose of a COVID-19 vaccine but did not receive a second dose is high. Vaccination timeliness remains an issue in the covid-19 vaccination process. Most persons who received the first dose of covid-19 vaccine haven't received their second dose after 21 days for the Sinopharm vaccines. This percentage has an increase trend varying from 57.6\% for the period April 12 to May 3, 2021 to $96.4 \%$ for the period $20^{\text {th }}$ April to $11^{\text {th }}$ May 2000.

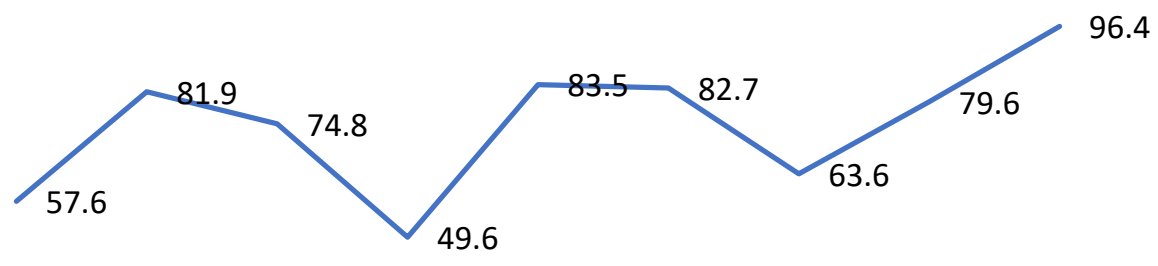

12april 13april 14april 15april 16april 17april 18april 19 april 20 april
to 3 may to 4 may to 5 may to 6 may to 7 may to 8 may to 9 may $\begin{gathered}\text { to } \\ 10 \text { may }\end{gathered}$ tomay

Figure 5: Percentage of persons who received first dose covid-19 vaccine but did not come for the second dose after 21 days.

\section{CHALLENGES}

The deployment of the COVID-19 vaccine is a dynamic process and this preliminary study provides an overview of the challenges Cameroon is currently facing. Three key challenges emerged from the actual experience in the first weeks of the COVID-19 vaccination efforts namely logistics, financing, and communication. 


\section{- The risk of great number of vaccines expiring}

One of the greatest challenges facing the Government of Cameroon is a substantial number of vaccines near expiration date. Of the 391200 doses received a month ago 364180 doses remain which represents 93per cent to expired in July and August 2021. After an acceleration phase, the curb has plateau for the past 10 days. With simple arithmetic it is known that about 4,000 doses have to be administered every day in order to use the vaccines. With the extremely low number of people vaccinated, the likelihood of a great stock of vaccines that are going to expire is great.

\section{- Vaccine hesitancy, and its Spillover effect on mass vaccination campaigns}

On Tuesday, April 27, 2021 a contradictory debate was held as part of the activities of the African Immunization Week on the theme "Overcoming vaccine hesitancy in Cameroon". This round table brought together eminent personalities, local elected officials, members of the media, bloggers and influencers, the officials of the ministry of Health, the directors of hospitals, the media, the technical and financial partners and the leaders of civil society It emerged from the round table that vaccine hesitation was accentuated with the arrival of the vaccine against Covid-19 and especially linked to certain peculiarities: the speed of marketing of this vaccine; the diversity of the vaccines (many laboratories involved); the high political involvement with the COVID19 vaccine in particular which according to some Cameroonian makes it even more suspicious; some of the participants thought that the targets of the vaccines are guinea pigs for pharmaceutical companies whose mercantilism is very often above the well-being of individuals. And for those who oppose the inoculation of any vaccine to protect themselves from disease, not all the truths are told.

In addition, the covid 19 context and its rumors, had a negative impact on ongoing mass vaccination campaigns in Cameroon. During the national immunization days for the vaccination campaign against polio that started across the country on May 7, 2021 and ended on the 9th of the same month, targeting children under 5 years old, but a significant proportion of the population refused to take the Oral Polio Vaccines thinking the government was forcing covid 19 vaccines on them; Unlike the Covid-19 vaccine, OPV is administered orally, but a certain misunderstanding has sowed confusion in the minds which prevented a successful development of this operation in several regions of the country. The population believed that the National immunization days was a pretext for administering the vaccine against the Covid-19 to the populations and even more so to children. 


\section{- Implementation in emergency mode, Putting the horse before the plow}

Implementation in emergency mode has offered little time for scaling up communication; New vaccine's introduction requires significant preparation and structural complexity to ensure optimal vaccine delivery. One month after the start of the campaign the communication strategy is not yet deployed and the risk communication plan not validated neither implemented. The microplanning is not yet done at the level of health centers. Micro-planning is one of the tools used by health workers to identify priority communities, to overcome existing barriers and to develop work plans leading to solutions. it indicates how to plan immunization sessions and organize follow-up for "lost to follow-up". Micro-planning is not yet done one month after the launch of the vaccination which has left health centers unprepared and all the aspect including communication not fully done The population felt that the Expanded Immunization Program did not communicate enough and long in advance. Some key opinion leaders complained that they did not know how the vaccines looked like but it was already been administered. It might be difficult to achieve success when the basics are not covered.

\section{- Cumbersome disbursements procedures}

Cumbersome financial and administrative procedures have made it difficult to respond immediately to urgent needs leading to a delay in setting up the technical assistance. The delays in disbursements led to underfunding of preparatory activities which in turn has led to a delay in setting up the minimum conditions for introduction; in addition the Small quantity of vaccine allocation that is 600 hundred thousands of doses to be distributed nationally has generated high operational cost. Finally, ensuring long-term financing of operational costs, integrating the vaccination against Covid19 into routine activities while ensure long-term financing of operational costs remains a daunting challenge:

\section{LESSONS LEARNED}

Despite these several challenges, some approaches used to increase vaccine uptake of COVID19 vaccines could be instructive, and could be applied to many countries around the world, especially low-income countries.

\section{- Political leadership}


The involvement of the Prime Minister, all the Ministers traditional authorities lilke Sultans Lamidos, Governors, Heads of hospitals have all received their jab in front of the media and their COVID-19 vaccination zas broadcasted live on national television. This gesture has within just two days of the launch created a momentum around and influenced positively those around them to get vaccinated

\section{- Intensive 5-day vaccination campaign}

The intensive 5-day vaccination campaign started on April 26, 2021 which brought a tenfold increase compare to non-campaign day and improved the overall uptake. During these intensive vaccination campaigns mobile teams and sites were increased, spaces have been set up to house vaccination points in order to facilitate the population's access to the administration of the vaccine. The daily trends analysis showed that during the intensive vaccination campaigns the daily doses administered were high and has started dwindling at the end of the campaign. Achieving high immunization coverage through intensive vaccination campaigns is a best practice that needs to be renewed and scale up.

\section{- Invest in early proactive communication}

A month after starting the vaccination communication and demand generation for COVID-19 vaccines has been very slow, centralized and, weak. As such communications must start early to prepare communities to receive the vaccine. Introduction of new vaccines follows stringent protocols with an overdose of communication which has not been the case during the hasty introduction of COVID19 vaccines. The good intention to avoid delays in the implementation and the start of vaccination to give the protection to those who need them the most may have hurt the communication and may be the trust. Consumer habits have been fundamentally transformed and are essentially geared towards immediacy and proximity. Local communication needs to start early enough to reach customers and prospects in specific catchment area. It is imperative to make accurate information available to the populations, to build a climate of trust with them. This will make it possible to avoid rumors and to dispel the fear cultivated by certain manipulators.

\section{DISCUSSION}

If the country does not reach high levels of vaccination, the virus is likely to continue circulating in pockets particularly with women representing only one third of all vaccinated; The national coverage rate is 5.4 percent at the national level to date hides disparities at the regional 
level. Immunization coverage is low overall but lower for women. A figure which does not however reflect the Cameroonian population composed of $48 \%$ men and 52\% women; in addition, women are also more represented within health professionals. It is therefore important to study variations in immunization coverage according to income and geographic location, to understand the reasons why COVID19 Vaccination coverage is low for women, and how gender roles and activities can prevent women's immunization.

Given the newness of the COVID vaccines, the display of AEFI will not be fully known until the vaccines are used widely; evaluation of adverse events following immunizations, (AEFI) remains necessary to provide a more complete benefit-risk profile; Ongoing surveillance for the potential emergence of adverse effects side-effect monitoring is necessary to overcome vaccine hesitancy. Moreover, the majority do not show up on time for their second doses it is important to follow this indicator closely to know whether it is just a delay in getting the second dose or a dropout and understand the reasons behind both phenomenon's.

Given the high likelihood of not being able to administer all the doses of vaccines strategies to increase the uptake and reverse the trends may include, to increase the number of vaccination centers, in order to make the service closer to the population., expand the target population. It is of outmost importance to involve just any voluntary adult. The COVID-19 vaccination in Cameroon was initiated with two types of vaccines: Covishield and Sinopharm. Later on, the country plans to order 4 million doses of COVID vaccine from the Johnson and Johnson laboratory and 8 thousand doses of Sputnik: The availability of different types of COVID-19 vaccines represents an opportunity to mitigate the effects of the global pandemic.

. The conclusions of this paper are subject to some limitations because the analysis relied on preliminary and aggregated data and could be subject to delays in reporting. It gives an early overview of the challenges, the achievements and the lessons learned as a contribution to significant discussions on strategies to improve service delivery and uptake. The two main obstacles remain the lack of funding and the weak activity of communication due to slow disbursement of funds. It is urgent to implement effective communication and strong community engagement to save routine vaccination and make the current vaccination campaign against Covid19 a success. 


\section{AUTHOR CONTRIBUTIONS}

A.A, AB , and D.D. designed the experiment/survey instrument and conducted the analysis. A.A contributed to the conceptual design of the research and A.A and D.D to the writing of the paper.

\section{Competing interests}

The authors declare no competing interest

\section{Acknowledgements}

This study received no specific funding or grant from any agency in the public, commercial, or not-for-profit sectors.

\section{REFERENCES}

1. https://worldpopulationreview.com/countries/cameroon-population

2. Ministry of Public Health. Covid-19 Preparation And Response Plan In Cameroon, Feb 2020

\section{3. https://www.cameroon-tribune.cm/article.html/39253/fr.html/commitment-taken- promised-fulfilled}

4. Ministry of Public Health. Response Plan To The Covid-19 Epidemic In Cameroon For The Health Sector For 2021 / Ministry of Public Health, COVID-19 preparedness and response plan DRAFT, March 2021

5. Ministry of Public Health, Study on the acceptability and logic of the populations for the introduction of the COVID-19 vaccine in Cameroon, 2021.

6. Dinga, J. N., Sinda, L. K., \& Titanji, V. P. K., Assessment of Vaccine Hesitancy to a COVID-19 Vaccine in Cameroonian Adults and Its Global Implication. Vaccines, 9 (2), 2021. https://doi.org/10.3390/vaccines9020175

7. Ministry of Public Health. National Communication Plan for the deployment of vaccination against COVID-19 in Cameroon, March 202

8. Plan de communication des risques et d'engagement communautaire contre l'épidémie de covid-19 au Cameroun Avril 2021 
9. Ndipowa et al Don .J.Infect. Diseas .Immun. 008 Donnish Journal of Infectious Diseases and Immunity Vol 2(2) pp. 007-010 August, 2016. http://www.donnishjournals.org/djidi ISSN: 2984-8776 Copyright (C) 2016 Donnish Journals 